\title{
Stem cell signaling as a target for novel drug discovery: recent progress in the WNT and Hedgehog pathways
}

\author{
Songzhu Michael $\mathrm{AN}^{1, *}$, Qiang Peter DING ${ }^{1}$, Ling-song $\mathrm{LI}^{2}$ \\ ${ }^{1}$ Curegenix Inc, Guangzhou 510663, China; '2Shanghai Advanced Research Institute, Chinese Academy of Sciences, Shanghai 201203, \\ China
}

\begin{abstract}
One of the most exciting fields in biomedical research over the past few years is stem cell biology, and therapeutic application of stem cells to replace the diseased or damaged tissues is also an active area in development. Although stem cell therapy has a number of technical challenges and regulatory hurdles to overcome, the use of stem cells as tools in drug discovery supported by mature technologies and established regulatory paths is expected to generate more immediate returns. In particular, the targeting of stem cell signaling pathways is opening up a new avenue for drug discovery. Aberrations in these pathways result in various diseases, including cancer, fibrosis and degenerative diseases. A number of drug targets in stem cell signaling pathways have been identified. Among them, WNT and Hedgehog are two most important signaling pathways, which are the focus of this review. A hedgehog pathway inhibitor, vismodegib (Erivedge), has recently been approved by the US FDA for the treatment of skin cancer, while several drug candidates for the WNT pathway are entering clinical trials. We have discovered that the stem cell signaling pathways respond to traditional Chinese medicines. Substances isolated from herbal medicine may act specifically on components of stem cell signaling pathways with high affinities. As many of these events can be explained through molecular interactions, these phenomena suggest that discovery of stem cell-targeting drugs from natural products may prove to be highly successful.
\end{abstract}

Keywords: stem cells; signaling pathway; WNT; Hedgehog; cancer; fibrosis; cardiac remodeling; osteoporosis; traditional Chinese medicine; drug discovery

Acta Pharmacologica Sinica (2013) 34: 777-783; doi: 10.1038/aps.2013.64; published online 27 May 2013

\section{Introduction}

Over the past 30 years, developmental biology studies have revealed key signal transduction processes involved in embryonic development. Signals elicited by the extracellular WNT, Hedgehog, Notch, fibroblast growth factors (FGF), and transforming growth factor (TGF)- $\beta$ proteins are the most important events. Through intracellular signaling cascades, these secreted proteins transduce signals to the recipient stem cells or their differentiated progeny. As a result, the stem cells or differentiated cells undergo cellular phenotypic changes in self-renewal, differentiation, proliferation or migration. The consequences of these signaling events lead to changes at the organism level, which are manifested as the development of the embryo or as physiological functions in adults. Among these signaling pathways, the WNT and Hedgehog signaling

\footnotetext{
* To whom correspondence should be addressed.

E-mail man@curegenix.com

Received 2013-02-06 Accepted 2013-04-12
}

pathways are perhaps the most essential to the development of all metazoans. From decades of genetic research, the key components of the WNT and Hedgehog signaling cascades and the mechanisms by which these signals act in embryonic development have been well-established. By contrast, our understanding of how these molecules maintain homeostasis and regeneration in adults is still limited. The advances in stem cell research and chemical biology have now provided an opportunity to investigate these pathways in a broad range of biological systems and disease models. In particular, there are now unique experimental opportunities to use chemical modulators of the WNT and Hedgehog pathways to explore disease mechanisms and the therapeutic utility of these modulators.

\section{The WNT pathway}

The WNT genes were first discovered in Drosophila genetic studies $^{[1]}$ and then later in cancer biology as the mouse oncogene int- ${ }^{[2]}$ (Figure 1). In humans, the WNT proteins are a 

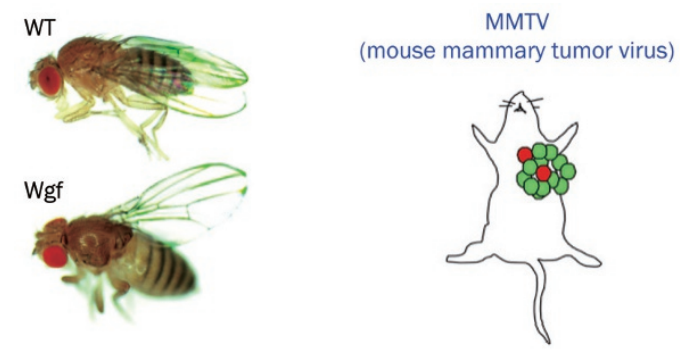

Figure 1. The discovery of the WNT genes in fly (Wingless) and mouse (int-1) models. Left: the Wingless phenotype in Drosophila; right: mouse breast tumor formation induced by MMTV insertion into the mouse int-1 locus. Wingless and int-1 are homologous to each other, and the name Wnt was coined by combining the two words, Wingless and int-1.

family of 19 lipidated and glycosylated proteins that play essential roles in diverse processes such as stem cell selfrenewal, differentiation, cell polarity, proliferation and migration (The WNT homepage: http: //www.stanford.edu/ group/nusselab/cgi-bin/wnt/). Perturbations in the levels or activities of the WNT proteins or of any of the signaling components in the WNT pathway result in changes in embryonic development or adult physiology ${ }^{[3]}$.

The WNT proteins modulate both the canonical ( $\beta$-catenindependent) and the non-canonical ( $\beta$-catenin-independent) WNT signaling pathways. The canonical WNT signaling pathway stabilizes $\beta$-catenin and, thus, the transcription of genes known to regulate a vast array of cellular functions (Figure 2). The non-canonical WNT signaling pathway does not activate $\beta$-catenin but instead transduces signals to affect cell polarity and migration through the planar cell polarity pathway and small GTPases.

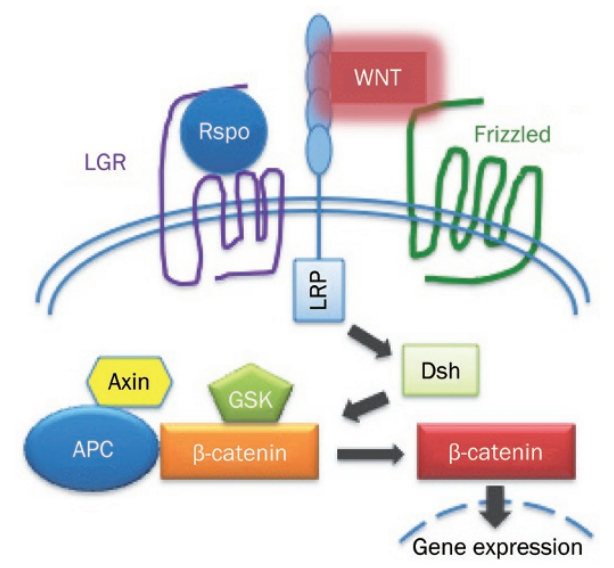

Figure 2. A diagram of the WNT pathway.

\section{The Hedgehog pathway}

The Hedgehog gene was also first discovered in Drosophila genetics $^{[1]}$. In vertebrates, the Hedgehog proteins are a family of three lipidated proteins (Sonic, Desert and Indian Hedge- hog) that play essential roles in diverse processes, such as stem cell self-renewal, differentiation, and proliferation ${ }^{[4]}$. Hedgehog proteins act through their receptors to transduce signals inside the cells, resulting in the regulation of embryonic development or adult physiology ${ }^{[5]}$.

Hedgehog signaling also consists of canonical and noncanonical pathways ${ }^{[6]}$. The canonical pathway, shown in Figure 3, transduces Hedgehog signaling to the Gli family of transcription factors, which regulate the transcription of genes involved in a vast array of cellular functions ${ }^{[4]}$. The noncanonical Hedgehog signaling pathway does not involve Gli, but rather it transduces signals via the calcium-dependent activation of AMPK, which triggers rapid Warburg-like metabolic reprogramming and is required for proper metabolic selectivity and flexibility in muscle and fat ${ }^{[7]}$.

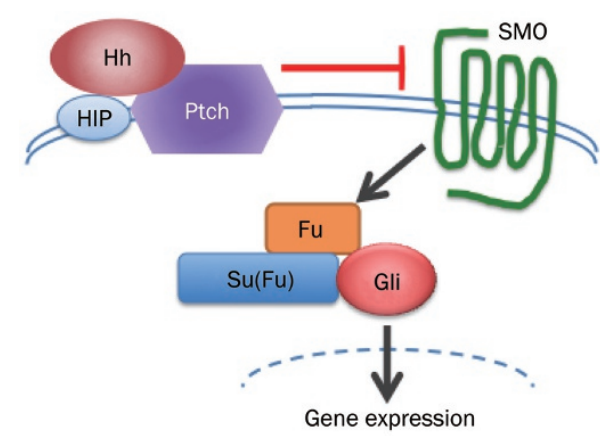

Figure 3. A diagram of the Hedgehog (Hh) pathway.

\section{Stem cell signaling pathways in diseases}

Given the importance of stem cell signaling pathways in embryonic development, it is not surprising that genetic mutations that perturb pathway activities result in growth abnormalities in humans. Because many physiological homeostasis activities and pathological responses in adults are related to stem cell functions, aberrations in the pathways that regulate stem cells result in diseases. Several human diseases, especially those associated with tissue damage and repair, such as cancer, fibrosis, degenerative diseases and skeletal repairs, are the most relevant ${ }^{[3]}$.

\section{The Wnt pathway in cancer}

A possible role for the WNT pathway in cancer was first discovered in the 1980s in mouse models of breast cancer. Aberrant over-expression of WNT1, induced by a viral insertion at the Wnt1 locus (also known as int1), causes spontaneous mammary hyperplasia and tumors in mice ${ }^{[8]}$. Further studies found that both WNT1 and other WNTs promoted the stabilization of free pools of $\beta$-catenin (CTNNB1) and the activation of CTNNB1-dependent transcription ${ }^{[9]}$. In the 1990s, several studies pointed to a crucial role for hyperactivated WNTCTNNB1 signaling in colorectal cancers ${ }^{[10,11]}$. Inherited inactivating mutations in adenomatous polyposis coli (APC) are found in patients with familial adenomatous polyposis (FAP), 
which can progress to colorectal carcinomas. These observations established that the unregulated activation of the WNT pathway is the most important oncogenic event in colorectal tumorigenesis ${ }^{[12]}$. Strikingly, activating CTNNB1 mutations or inactivating APC mutations occur in more than $80 \%$ of patients. A recent study revealed another mechanism by which $10 \%$ of colorectal tumors activate the WNT pathway; in this case, two genes encoding enhancers of the WNT ligands R spondin- 2 and $\mathrm{R}$ spondin- 3 are transcriptionally activated by fusion to other genes ${ }^{[13]}$.

In addition to colorectal cancer, several other types of cancer carry mutations in the WNT pathway. For example, liver cancer has approximately a $25 \%$ chance of gaining activating mutations in CTNNB1 or APC, while gastric cancer has approximately a $20 \%$ chance and lung cancer has approximately a $2 \%$ chance of gaining such mutations (Sanger Cancer Genome Project database, http: //www.sanger.ac.uk/genetics/CGP/). Liver cancer and gastric cancer, along with colorectal cancer, are some of the most prevalent types of cancer in China.

Another aspect of cancer development involving the WNT pathway is metastasis. Several recent studies have suggested that WNT signaling plays a critical role in the development of metastasis. Nguyen and colleagues showed that the activation of the canonical WNT/TCF pathway is a determinant of metastasis to brain and bone during lung adenocarcinoma progression $^{[14]}$. In addition, Haber and colleagues showed that circulating tumor cells (CTCs) that are shed from pancreatic tumors express WNT2, which suppresses anoikis, enhances anchorage-independent sphere formation, and increases the metastatic propensity in vivo. The formation of non-adherent tumor spheres by pancreatic cancer cells is associated with the upregulation of multiple WNT genes, and pancreatic CTCs displayed an enrichment for WNT signaling in 5 out of 11 cases. These analyses identified the WNT pathway as a candidate therapeutic target to prevent the distal spread of cancer ${ }^{[15]}$. The successful initiation of metastatic growth (metastatic colonization) is also linked to WNT signaling. Malanchi and colleagues demonstrated that a small population of cancer stem CSCs is critical for metastatic colonization because the initial expansion of cancer cells at the secondary site interact with the stromal niche signals. They found that periostin (POSTN), a component of the extracellular matrix, is expressed both in the stroma of the primary tumor and by fibroblasts in the normal tissue. The role of POSTN in cancer stem cell maintenance and metastasis is to recruit Wnt proteins and thereby increase WNT signaling in CSCs ${ }^{[16]}$.

The relationship between the WNT pathway and metastasis is not surprising given the importance of WNT signaling in mesenchymal development and the epithelial-mesenchymal transition (EMT) during embryonic development. EMT and the reverse mesenchymal-epithelial transition (MET) are analogous between development and tumor metastasis ${ }^{[17]}$.

\section{The Wnt pathway in fibrosis}

Recent investigations implicate WNT signaling in abnormal wound repair that leads to fibrosis. In patients with fibrotic diseases, there is elevated expression in components of the pathway. In animal models, the activation of the WNT canonical signaling pathway is responsible for injury repair that leads to fibrogenesis ${ }^{[18]}$.

Several types of fibrosis have been linked to the WNT pathway. For example, idiopathic pulmonary fibrosis (IPF) patients display aberrant activation in the $\mathrm{WNT} / \beta$-catenin signaling pathways in the lungs ${ }^{[19]}$. Additionally, it was found that significant increases in the nuclear levels of $\beta$-catenin occur in fibroblasts in the skin of systemic sclerosis patients compared to fibroblasts in the skin of healthy individuals. It was further shown that the nuclear accumulation of $\beta$-catenin has direct implications for the development of fibrosis in mice with fibroblast-specific stabilization of $\beta$-catenin. In contrast, fibroblast-specific deletion of $\beta$-catenin significantly reduced bleomycin-induced dermal fibrosis ${ }^{[20]}$.

A link between the canonical WNT pathway and a wellknown fibrogenic pathway, TGF- $\beta$ pathway, has been recently reported. While the activation of the canonical Wnt pathway stimulates fibroblasts in vitro and induces fibrosis in vivo, TGF- $\beta$ stimulates canonical WNT signaling by decreasing the expression of the WNT antagonist DKK-1. Transgenic overexpression of DKK-1 ameliorates skin fibrosis induced by constitutively active TGF- $\beta$ receptor signaling. These findings not only demonstrated that the canonical WNT pathway is necessary for TGF- $\beta$-mediated fibrosis but also identified a novel interaction between two key pathways in fibrosis ${ }^{[21]}$.

\section{The Wnt pathway in cardiac remodeling after acute myocardial infarction}

As an important regulator of differentiation and morphogenesis that controls stem cell fates, the WNT pathway is one of the important signals that forms the heart. Indeed, the organogenesis of the heart is tightly controlled by WNT signaling ${ }^{[22]}$.

This knowledge has been used in the induction of mesoderm tissue and subsequent cardiac differentiation from human ES cells in culture by using modulators of the WNT pathway. In the early phase, activation of canonical WNT signaling enhances mesoderm induction, while the later cardiac differentiation requires the inhibition of the canonical signal. This biphasic control of the WNT pathway permits the efficient generation of cardiomyocytes from human ES or iPS cells, and modulators of WNT signaling have been postulated as useful tools or drugs for basic studies or cardiac repair applications ${ }^{[23,24]}$.

Upon myocardial infarction, the heart reactivates several signaling pathways involved in cardiogenesis in an attempt to regenerate itself. It has been shown that the inhibition of canonical WNT signaling significantly reduces post-infarction mortality and functional decline. In addition, WNT signaling is implicated in the process of left ventricular (LV) remodeling by soluble frizzled-related proteins (sFRPs) that block the WNT-dependent activation of the canonical WNT pathway. In animal studies, sFRPs injected into the heart attenuated LV remodeling. Notably, sFRPs are secreted from bone marrow- 
derived mononuclear cells, which may serve as a mechanism for the therapeutic action of such cells in human heart failure patients ${ }^{[25]}$.

The cellular mechanism by which WNT signaling is involved in cardiac remodeling processes may be related to its role in fibrosis. As mentioned above, the WNT pathway plays a key role in the fibrosis of various organs. Much remains to be discovered about the role of WNT signaling before its therapeutic potential in heart failure can be more effectively explored $^{[26]}$.

\section{The Wnt pathway in bone diseases}

Another class of diseases where WNT signaling plays an important role is the skeletal diseases, such as osteoporosis. This connection once again stems from the involvement of this pathway in embryonic development, during which WNT signaling plays a key role in the development of bone, cartilage and muscle. For example, inactivating mutations of the SOST gene cause a reduction in sclerostin levels and are associated with high bone mass and unusual skeletal strength. The demonstration of sclerostin as an inhibitor of the WNT pathway quickly led to the development of an anti-sclerostin antibody for the treatment of osteoporosis ${ }^{[27]}$.

\section{The Hedgehog pathway in cancer}

A direct link between Hedgehog signaling and cancer was discovered in the 1990s. Activating mutations in two key Hedgehog signaling components, Patched (PTCH), and Smoothened (SMO), were discovered in medulloblastomas and basal cell carcinomas ${ }^{[28,29]}$. Since then, attempts have been made to identify links between the pathway and other more prevalent types of cancer. However, it appears that the activation of Hedgehog signaling in tumor cells by mutational events is limited, although paracrine activation of Hedgehog signaling in the tumor stroma appears to be a more dominant mechanism ${ }^{[30]}$.

\section{The WNT and Hedgehog pathways in CSCs}

Tumor relapse and metastasis remain major obstacles in cancer treatments. The hypothesis that tumors are maintained by dedicated stem cells, the cancer stem cell hypothesis, has attracted great interest. The purported cancer CSCs or tumorinitiating cells (TICs) are thought to have similar properties to those of stem cells, including the ability to self-renew, to form differentiated progenies and to develop resistance to chemotherapy. It is reasonable to postulate that CSCs employ many of the same signaling pathways found in normal stem cells, such as the WNT, Hedgehog and Notch pathways ${ }^{[31]}$.

Zhao et al showed that the loss of the Hedgehog pathway component SMO decreases the induction of chronic myelogenous leukemia (CML) by BCR-ABL. Loss of SMO causes the depletion of CML stem cells, whereas constitutively active SMO augments the CML stem cell number and accelerates the disease. These data indicate that the Hedgehog pathway is required for the maintenance of hematopoietic CSCs ${ }^{[32]}$.

The origin of CSCs is not fully understood, but recent data suggest that they can originate from normal stem or progeni- tor cells that live long enough to accumulate the multiple genetic changes necessary to initiate a tumor. In a mouse model, Schepers and colleagues provided direct functional evidence for the presence of stem cell activity within primary intestinal adenomas, which are precursors to intestinal cancer. By "lineage retracing", they demonstrated that the crypt stem cell marker Lgr5 marks a subpopulation of adenoma cells that fuel the growth of established intestinal adenomas. Notably, Lgr5 is a direct target gene of the canonical WNT pathway, which is expressed in normal gut stem cells in the crypt ${ }^{[33]}$. A recent study that used a genetic model of intestinal epithelial cell (IEC)-restricted constitutive WNT pathway activation demonstrated that WNT signaling that was induced by NF-KB signaling induced the de-differentiation of non-stem cells that go on to acquire tumor-initiating capabilities ${ }^{[34]}$.

\section{Drug discovery for the WNT and Hedgehog pathways}

Drug discovery based on the WNT and Hedgehog pathways has been one of the most active areas in the drug discovery field over the past decade. Many pharmaceutical companies and research institutes have been actively searching for both small molecule and large molecule drugs targeting these pathways. Recently, remarkable progress has been made in the search for drugs for the WNT pathway, and a hedgehog pathway antagonist, vismodegib (Erivedge), has recently been approved by the US FDA for the treatment of skin cancer.

\section{Hedgehog pathway inhibitors of cancer}

The first small molecule inhibitor targeting SMO was discovered by the biotech company Curis ${ }^{[35]}$. In collaboration with Curis, Genentech/Roche developed GDC0449 for the treatment of advanced basal cell carcinoma of the skin. Clinical trial results showed dramatic efficacy of the drug vismodegib (Erivedge) in patients with metastatic basal cell carcinoma ${ }^{[36]}$. This led to the market approval of the drug by the US FDA in 2012, a milestone for drug development in this field. GDC0449 and several other SMO antagonists are currently being tested in clinical trials as potential treatments for medulloblastoma, pancreatic cancer, ovarian cancer and hematopoietic cancers.

\section{WNT pathway inhibitors of cancer}

Given the importance of the WNT pathway in many types of cancer, it is not surprising that the discovery of drugs targeting this pathway has been actively pursued by both the pharmaceutical and biotech industries. After a decade of failures, several promising programs have entered clinical trials over the past two years.

The most advanced clinical program is an anti-Frizzled antibody from OncoMed Pharmaceuticals that entered into Phase I clinical trials in 2011. Published data show that this panFrizzled antibody (OMP18R5) has remarkable anti-tumor efficacy in many preclinical patient-derived xenograft models ${ }^{[37]}$. OncoMed also has several other WNT pathway inhibitors in their pipeline, including a Frizzled-8 Fc fusion protein and an antibody against R-spondin/LGR5.

Also in clinical Phase I trials is a small molecule inhibitor of 
porcupine, an acetyl-transferase responsible for the lipidation, and hence the bioactivity, of all the WNT proteins. Developed by Novartis, LGK974 is being tested against multiple types of cancer in Phase I trials (http: / / clinicaltrials.gov/show/ NCT01351103).

Several WNT inhibitors have also been pursued in preclinical studies. For example, by screening for agents that specifically kill CSCs, Weinberg, Lander and colleagues discovered compounds that are selectively toxic to CSCs isolated from breast cancers. One compound, salinomycin, inhibits mammary tumor growth in vivo and induces increased epithelial differentiation of tumor cells ${ }^{[38]}$. A subsequent study by another group demonstrated that salinomycin is a potent inhibitor of the WNT signaling cascade and acts by interfering with LPR6 phosphorylation ${ }^{[39]}$. The compound was once pursued by the biotech company Verastem in preclinical development targeting breast cancer.

\section{Wnt pathway inhibitors for fibrosis}

Because aberrant activation of the WNT signaling cascade occurs in the lungs of patients with pulmonary fibrosis, targeting this pathway for intervention in IPF has been investigated. Henderson Jr and colleagues showed that ICG-001, a small molecule that specifically inhibits TCF/ $\beta$-catenin transcription in a CBP-dependent fashion, significantly attenuates bleomycin-induced lung fibrosis in mice. Because no effective treatment for IPF currently exists, the selective inhibition of canonical WNT signaling may become a potential unique therapeutic approach for pulmonary fibrosis ${ }^{[40]}$.

The role of WNT signaling in dermal fibrosis was investigated by targeting tankyrases as novel molecular targets for the inhibition of canonical WNT signaling. The anti-fibrotic effects of the tankyrase inhibitor XAV-939 in experimental fibrosis was shown by the effective reduction of bleomycininduced dermal thickening, the differentiation of resting fibroblasts into myofibroblasts and the accumulation of collagen. Thus, tankyrase-mediated canonical WNT signaling might be a potential target for therapy in fibrotic diseases ${ }^{[41]}$.

\section{Wnt pathway inhibitors for cardiac remodeling after myocardial infarction}

Young and coworkers showed that daily administration of pyrvinium, a small molecule inhibitor of WNT signaling and an FDA-approved anti-parasite drug, directly into the heart of mice with myocardial infarction (MI) reduced adverse cardiac remodeling ${ }^{[42]}$. Unfortunately, the underlying mechanism has not been well studied, and pyrvinium is not a suitable compound for heart failure therapy because of its toxicity. Nevertheless, these results encourage the testing of other WNT inhibitors in this disease model.

\section{Wnt pathway activators for osteoporosis}

Given the dramatic bone phenotype caused by the SOST mutations, the inhibitor of sclerostin quickly became a drug candidate for the treatment of osteoporosis, and a monoclonal antibody against sclerostin AMG785 has been developed by the biotech giant Amgen. Phase II results released by Amgen strongly suggest that this inhibitor holds promise for osteoanabolic therapy of osteoporosis ${ }^{[43]}$.

\section{TCM nature products targeting stem cell pathways}

Natural products, particularly Chinese herbal medicines, are rich sources of pharmacologically active substances. Identifying the active ingredients in traditional Chinese medicines (TCMs) has been an active area of research in the Chinese pharmacological community. Through our own investigations, we have discovered that the stem cell signaling pathways tend to be targeted by the natural products in TCMs. Many pharmacologically active substances isolated from plants or microbes specifically act on components of stem cell signaling pathways with reasonable affinities. For example, the Chinese herbal medicine Qingdai, which is derived from the plant Indigofera suffruticosa, contains a chemical called indirubin (Figure 4). As a component in a famous TCM formula Dong Gui Long Hui Wan, Qingdai has been used in China for years for the treatment of leukemia and other types of cancers. Notably, the active ingredient indirubin has been identified as an inhibitor of GSK-3 $\beta$, a key intermediate of the WNT pathway, and WNT signaling has been implicated in leukemias such as CML and other types of cancers. The Indigofera genus has more than 700 species, and different indirubins have been discovered in various plants in the genus and in marine organisms. It is logical to predict that novel pharmacologically active indirubins can be discovered using a WNT pathway screening assay.

Another example comes from cyclopamine, a steroidal alkaloid extracted from Veratrum. It has been shown that cyclopamine inhibits Hedgehog signaling by directly binding with SMO (Figure 5). Such a specific molecular interaction is perhaps not surprising given that the natural ligands for SMO are oxysterols $^{[44]}$. In TCMs, Veratrum has been used for many years, including its use as part of a special folk remedy for treating skin cancer. It would not be surprising to discover that the therapeutic effects of cyclopamine are caused by the inhibition of the Hedgehog pathway in the most common form of skin cancer, basal cell carcinoma. There are more than 80 different species of Veratrum plants in China, and it would be interesting to identify novel cyclopamine-like compounds that may serve as drug candidates or as origins for novel derivatives.

\section{Perspectives}

Stem cell research has great potential not only to enhance our understanding of basic biology but also to revolutionize the practice of medicine. In addition to cell therapy, the use of proven approaches to discover drugs that target stem cell signaling pathways offers a particularly exciting opportunity. Over the past few years, remarkable progress has been made in the search for drugs that target two of the most important stem cell pathways, the WNT and the Hedgehog pathways. The pharmaceutical industry has placed great expectations on a number of clinical candidates that target these pathways. 


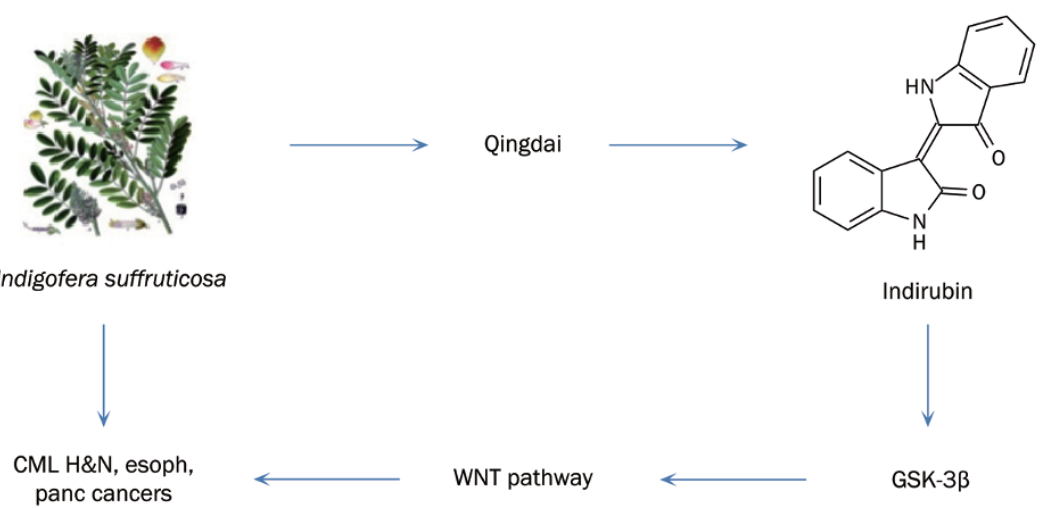

Figure 4. Indirubin, which can be derived from the Chinese herbal medicine Qingdai, is a potent inhibitor of GSK-3ß, a key intermediate in the WNT pathway.

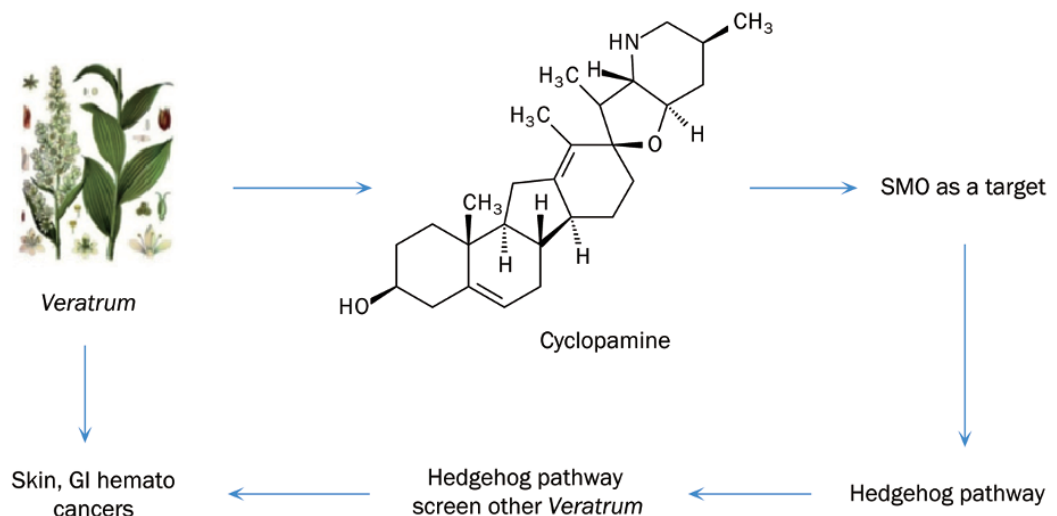

Figure 5. Cyclopamine, which can be derived from Chinese herbal medicine Veratrum, is a potent and specific inhibitor of SMO, a key intermediate in the Hedgehog pathway.

Of perhaps the most interest to the Chinese pharmaceutical community is the tendency of traditional Chinese medicines to target these stem cell signaling pathways. Many pharmacologically active substances isolated from plants or microbes act specifically on components of stem cell signaling pathways. As such events can be explained through specific molecular interactions, this phenomenon is non-random and suggests that the targeted discovery of stem cell-modulating drugs from natural products may prove to be highly successful ("druggability"). Many novel compounds can potentially be discovered by using stem cell signaling assays to screen the vast resources of medically active herbal medicines in China.

\section{References}

1 Nüsslein-Volhard C, Wieschaus E. Mutations affecting segment number and polarity in Drosophila. Nature 1980; 287: 795-801.

2 Rijsewijk F, Schuermann M, Wagenaar E, Parren P, Weigel D, Nusse R. The Drosophila homolog of the mouse mammary oncogene int-1 is identical to the segment polarity gene wingless. Cell 1987; 50 : 649-57.

3 Clevers H, Nusse R. Wnt/ $\beta$-catenin signaling and disease. Cell 2012; 149: 1192-205.
4 Ingham PW, Nakano Y, Seger C. Mechanisms and functions of Hedgehog signaling across the metazoa. Nat Rev Genet 2011; 12: 393-406.

5 Robbins DJ, Fei DL, Riobo NA. The Hedgehog signal transduction network. Sci Signal 2012; 5: re6.

6 Jenkins D. Hedgehog signaling: emerging evidence for non-canonical pathways. Cell Signal 2009; 21: 1023-34.

7 Teperino R, Amann S, Bayer M, McGee SL, Loipetzberger A, Connor T, et al. Hedgehog partial agonism drives Warburg-like metabolism in muscle and brown fat. Cell 2012; 151: 414-26.

8 Nusse R, Varmus HE. Many tumors induced by the mouse mammary tumor virus contain a provirus integrated in the same region of the host genome. Cell 1982; 31: 99-109.

9 Papkoff J, Rubinfeld B, Schryver B, Polakis P. Wnt-1 regulates free pools of catenins and stabilizes APC-catenin complexes. Mol Cell Biol 1996; 16: 2128-34.

10 Korinek V, Barker N, Morin PJ, van Wichen D, de Weger R, Kinzler KW, et al. Constitutive transcriptional activation by a $\beta$-catenin-Tcf complex in $\mathrm{APC}^{-/-}$colon carcinoma. Science 1997; 275: 1784-7.

11 Morin PJ, Sparks AB, Korinek V, Barker N, Clevers H, Vogelstein B, et al. Activation of $\beta$-catenin-Tcf signaling in colon cancer by mutations in $\beta$-catenin or APC. Science 1997; 275: 1787-90.

12 Kinzler KW, Vogelstein B. Lessons from hereditary colorectal cancer. Cell 1996; 87: 159-70. 
13 Seshagiri S, Stawiski EW, Durinck S, Modrusan Z, Storm EE, Conboy $\mathrm{CB}$, et al. Recurrent R-spondin fusions in colon cancer. Nature 2012; 488: 660-4.

14 Nguyen DX, Chiang AC, Zhang XH, Kim JY, Kris MG, Ladanyi M, et al. WNT/TCF signaling through LEF1 and HOXB9 mediates lung adenocarcinoma metastasis. Cell 2009; 138: 51-62.

15 Yu M, Ting DT, Stott SL, Wittner BS, Ozsolak F, Paul S, et al. RNA sequencing of pancreatic circulating tumour cells implicates WNT signaling in metastasis. Nature 2012; 487: 510-3.

16 Malanchi I, Santamaria-Martínez A, Susanto E, Peng H, Lehr HA, Delaloye JF, et al. Interactions between cancer stem cells and their niche govern metastatic colonization. Nature 2011; 481: 85-9.

17 Yang J, Weinberg RA. Epithelial-mesenchymal transition: at the crossroads of development and tumor metastasis. Dev Cell 2008; 14: 818-29.

18 Lam AP, Gottardi CJ. $\beta$-Catenin signaling: a novel mediator of fibrosis and potential therapeutic target. Curr Opin Rheumatol 2011; 23 : 562-7.

19 Königshoff M, Balsara N, Pfaff EM, Kramer M, Chrobak I, Seeger W, et al. Functional Wnt signaling is increased in idiopathic pulmonary fibrosis. PLoS One 2008; 3: e2142.

20 Beyer C, Schramm A, Akhmetshina A, Dees C, Kireva T, Gelse K, et al. $\beta$-Catenin is a central mediator of pro-fibrotic Wnt signaling in systemic sclerosis. Ann Rheum Dis 2012; 71: 761-7.

21 Akhmetshina A, Palumbo K, Dees C, Bergmann C, Venalis P, Zerr $\mathrm{P}$, et al. Activation of canonical Wnt signaling is required for TGF- $\beta$ mediated fibrosis. Nat Commun 2012; 3: 735.

22 Tzahor E. Wnt/beta-catenin signaling and cardiogenesis: timing does matter. Dev Cell 2007; 13: 10-3.

23 Paige SL, Osugi T, Afanasiev OK, Pabon L, Reinecke H, Murry CE. Endogenous Wnt/beta-catenin signaling is required for cardiac differentiation in human embryonic stem cells. PLoS One 2010; 5: e11134.

24 Lian X, Hsiao C, Wilson G, Zhu K, Hazeltine LB, Azarin SM, et al. Robust cardiomyocyte differentiation from human pluripotent stem cells via temporal modulation of canonical Wnt signaling. Proc Natl Acad Sci U S A 2012; 109: E1848-57.

25 Bergmann MW. WNT signaling in adult cardiac hypertrophy and remodeling: lessons learned from cardiac development. Circ Res 2010; 107: 1198-208.

26 Dawson K, Aflaki M, Nattel S. Role of the Wnt-Frizzled system in cardiac pathophysiology: a rapidly developing, poorly understood area with enormous potential. J Physiol 2013; 591: 1409-32.

27 Costa AG, Bilezikian JP. Sclerostin: therapeutic horizons based upon its actions. Curr Osteoporos Rep 2012; 10: 64-72.

28 Raffel C, Jenkins RB, Frederick L, Hebrink D, Alderete B, Fults DW, et al. Sporadic medulloblastomas contain PTCH mutations. Cancer Res 1997; 57: 842-5.

29 Xie J, Murone M, Luoh SM, Ryan A, Gu Q, Zhang C, et al. Activating Smoothened mutations in sporadic basal-cell carcinoma. Nature 1998; 391: 90-2.
30 Yauch RL, Gould SE, Scales SJ, Tang T, Tian H, Ahn CP, et al. A paracrine requirement for hedgehog signaling in cancer. Nature 2008; 455: 406-10.

31 Takebe N, Harris PJ, Warren RQ, Ivy SP. Targeting cancer stem cells by inhibiting Wnt, Notch, and Hedgehog pathways. Nat Rev Clin Oncol 2011; 8: 97-106.

32 Zhao C, Chen A, Jamieson CH, Fereshteh M, Abrahamsson A, Blum $J$, et al. Hedgehog signaling is essential for maintenance of cancer stem cells in myeloid leukaemia. Nature 2009; 458: 776-9.

33 Schepers AG, Snippert HJ, Stange DE, van den Born M, van Es JH, van de Wetering $M$, et al. Lineage tracing reveals Lgr $5^{+}$stem cell activity in mouse intestinal adenomas. Science 2012; 337: 730-5.

34 Schwitalla S, Fingerle AA, Cammareri P, Nebelsiek T, Göktuna SI, Ziegler PK, et al. Intestinal tumorigenesis initiated by dedifferentiation and acquisition of stem-cell-like properties. Cell 2013; 152: 25-38.

35 Frank-Kamenetsky M, Zhang XM, Bottega S, Guicherit O, Wichterle H, Dudek $\mathrm{H}$, et al. Small-molecule modulators of Hedgehog signaling: identification and characterization of Smoothened agonists and antagonists. J Biol 2002; 1: 10.

36 Sekulic A, Migden MR, Oro AE, Dirix L, Lewis KD, Hainsworth JD, et al. Efficacy and safety of vismodegib in advanced basal-cell carcinoma. N Engl J Med 2012; 366: 2171-9.

37 Gurney A, Axelrod F, Bond CJ, Cain J, Chartier C, Donigan L, et al. Wnt pathway inhibition via the targeting of Frizzled receptors results in decreased growth and tumorigenicity of human tumors. Proc Natl Acad Sci U S A 2012; 109: 11717-22.

38 Gupta PB, Onder TT, Jiang G, Tao K, Kuperwasser C, Weinberg RA, et al. Identification of selective inhibitors of cancer stem cells by highthroughput screening. Cell 2009; 138: 645-59.

39 Lu D, Choi MY, Yu J, Castro JE, Kipps TJ, Carson DA. Salinomycin inhibits Wnt signaling and selectively induces apoptosis in chronic lymphocytic leukemia cells. Proc Natl Acad Sci U S A 2011; 108 : 13253-7.

40 Henderson WR Jr, Chi EY, Ye X, Nguyen C, Tien YT, Zhou B, et al. Inhibition of Wnt/beta-catenin/CREB binding protein (CBP) signaling reverses pulmonary fibrosis. Proc Natl Acad Sci U S A 2010; 107: 14309-14.

41 Distler A, Deloch L, Huang J, Dees C, Lin NY, Palumbo-Zerr K, et al. Inactivation of tankyrases reduces experimental fibrosis by inhibiting canonical Wnt signaling. Ann Rheum Dis 2012 Nov 12.

42 Saraswati S, Alfaro MP, Thorne CA, Atkinson J, Lee E, Young PP. Pyrvinium, a potent small molecule Wnt inhibitor, promotes wound repair and post-MI cardiac remodeling. PLoS One 2010; 5: e15521.

43 Padhi D, Jang G, Stouch B, Fang L, Posvar E. Single-dose, placebocontrolled, randomized study of AMG 785, a sclerostin monoclonal antibody. J Bone Miner Res 2011; 26: 19-26.

44 Corcoran RB, Scott MP. Oxysterols stimulate Sonic hedgehog signal transduction and proliferation of medulloblastoma cells. Proc Natl Acad Sci U S A 2006; 103: 8408-13. 